\title{
Aplicación del Diseño Social en una experiencia local orientada al mejoramiento de políticas públicas de envejecimiento poblacional, Valparaíso-Chile
}

\section{Resumen}

El envejecimiento poblacional a nivel global y local es una realidad que desafía a todas las disciplinas, incluyendo al diseño. La Región de Valparaíso posee la mayor proporción de personas $\geq$ de 60 años o Adultos Mayores (AM) del país. Entre las situacionesproblema que enfrenta este grupo poblacional, en especial aquellos que se encuentran en un entorno social con múltiples carencias y vulnerabilidades, está la malnutrición. Chile cuenta con un programa de alimentación complementaria orientado a los AM, sin embargo, no ha dado el resultado esperado. Este artículo describe y analiza una experiencia de diseño llevada a cabo en Valparaíso, en el contexto de un proyecto social que busca mejorar la eficacia del programa público de alimentación. La experiencia, metodológicamente, consistió en el diseño participativo de un sistema de preparaciones alimentarias saludables para adultos mayores autovalentes, contemplando, por una parte, la caracterización sociodemográfica, de salud, hábitos y preferencias alimentarias de un grupo de adultos mayores autovalentes y vulnerables socioeconómicamente y, por otra, la realización de talleres de diseño participativo, para construir colaborativamente el sistema de comunicación del proyecto: marca y etiqueta de los alimentos. Se entregaron, además, preparaciones alimentarias para ser degustadas y valoradas por los AM durante tres meses. La participación fue voluntaria con firma de consentimiento informado. Resultados: los atributos positivos de la marca y las etiquetas que más destacan los AM son los siguientes: el diseño novedoso, el nombre, los dibujos, los colores Ilamativos; complementariamente, los aspectos que se debieran ajustar, desde la perspectiva de los AM, son: letras más grandes y claras. Desde el análisis de la experiencia se identifican tres factores críticos para este nivel de diseño social: 1. El rol del diseñador en un "modelo social" de intervención profesional, 2. El rol del usuario-participante en el proceso de co-diseño, y 3 . El rol del diseño en el contexto social.
Rossana Bastías Castillo Doctora Diseño y comunicación Universidad de Valparaíso, Facultad de Arquitectura, Escuela de Diseño Valparaíso, Chile

Correo electrónico:

rossana.bastias@uv.c

(10) orcid.org/0000-0001-5499-9591 Google Scholar

Mariane Lutz Riquelme

Magíster en Nutrición Humana Universidad de Valparaíso, Facultad de Medicina, Escuela de Medicina. Viña del Mar, Chile. Correo electrónico: mariane.lutz@uv.cl 당 orcid.org/0000-0001-6665-6748 Google Scholar

Isabel Siefer Navas

Magister en Enfermería Geronto-geriátrica Universidad de Valparaíso, Facultad de Medicina, Escuela de Enfermería Viña del Mar, Chile.

Correo electrónico: isabel.siefer@uv.cl https://orcid.org/0000-0003-2457-1353 Google Scholar

Raúl Gaete Fernández

Especialista en Administración Hotelera y Gastronómica

Universidad de Valparaíso, Facultad de Cs. Económicas y Administrativas, Escuela de Adm. Hotelera y Gastronómica Viña del Mar, Chile.

Correo electrónico: raul.gaete@uv.cl () orcid.org/0000-0002-1915-7759 Google Scholar

Recibido: mayo 29 de 2019

Aprobado: octubre 29 de 2020

Palabras clave:

diseño participativo, diseño social, co-diseño, adulto mayor, envejecimiento poblacional, alimentación saludable. 


\section{Application of Social Design in a local experience oriented to the improvement of public policies on population aging, Valparaíso-Chile}

\begin{abstract}
Global and local population aging is a reality that challenges all disciplines including design. The Valparaiso Region has the highest proportion of people $\geq 60$ years of age or Older Adults $(\mathrm{OA})$ in the country. Among the problem-situations faced by this population group, especially those who are in a social environment with multiple deficiencies and vulnerabilities, is malnutrition. Chile has a complementary feeding program oriented to the OA. However, it has not yielded the expected results. This article describes and analyzes a design experience carried out in Valparaiso in the context of a social project that seeks to improve the effectiveness of the public feeding program. Methodologically, the experience consisted of the participatory design of a system of healthy food preparations for self-sufficient older adults, considering, on the one hand, the socio-demographic, health, habits and food preferences characterization of a group of selfsufficient older adults and, on the other hand, the development of participatory design workshops to collaboratively build the communication system of the project: brand and food label. In addition, food preparations were delivered to be tasted and valued by the OAs for three months. Participation was voluntary with the signing of an informed consent form. Results: the positive attributes of the brand and labels most highlighted by the OAs are the following: the novel design, the name, the drawings, the eye-catching colors; in addition, the aspects that should beadjusted, from the perspective of the OAs, are: larger and clearer letters. From the analysis of the experience, three critical factors for this level of social design are identified: 1) The role of the designer in a "social model" of professional intervention; 2) the role of the user-participant in the co-design process; and 3) the role of design in the social context.
\end{abstract}

Key words:

participatory design, social design, co-design, elderly, aging population, healthy eating. 


\section{Introducción}

Un aspecto clave de la sociedad actual, tanto a nivel global como, en especial, a nivel local, es el proceso de transición demográfica que experimenta. Esta transición se refleja en un aumento abrupto en la proporción de población $\geq$ de 60 años o adultos mayores (AM), lo que plantea un gran desafío público para generar mecanismos de protección social a este grupo poblacional —en general- y de aquellos más vulnerables socioeconómicamente - en particular - (Apella, I. et al, 2019). Escenario de transformaciones que trae como correlato nuevos desafíos disciplinarios y profesionales para el diseño.

En su informe "Perspectivas de la Población Mundial", la Organización de las Naciones Unidas (ONU) (2017) estableció que la población de AM se duplicará en 2050, pasando de 962 millones en 2017 a más de 2100 millones al 2050. Asimismo, la Organización Mundial de la Salud (OMS) Ilama a los países miembros a realizar un cambio de visión de los AM, no ya como una población frágil o como una carga para la sociedad, sino como personas que cada vez son más activas y buscan oportunidades para relacionarse socialmente como sujetos de derechos. Este cambio de paradigma debe ir acompañado con garantizar un envejecimiento saludable; por esto la OMS también recomienda alinear los sistemas de salud con las necesidades de los AM, establecer sistemas para garantizar la atención crónica, crear entornos adaptados para el desarrollo y comprensión de este grupo de la población (OMS, 2015).

Como señala la Comisión Económica para América Latina y el Caribe (CEPAL), "estas nuevas configuraciones llevarán a nuevas necesidades y aspiraciones sociales, económicas, políticas y culturales de los diferentes grupos etarios, que deberán abordarse y resolverse mediante políticas públicas que garanticen la inclusión social y el pleno goce de derechos para todos" (CEPAL, 2017, p. 156). En este contexto Welti establece que se hace necesario que 
"el Estado implemente políticas sociales que atiendan las necesidades población" (Welti, 2013, p. 26), la salud de la población debiera ser un derecho reconocido por el sector público y no un "producto comercializable" (Marmot et al., 2008, p. 1667).

En Chile, el último censo de población realizado en 2017 registró 2.850.171 personas $\geq$ de 60 años, que representan el 16,22\% de la población total. En la Región de Valparaíso se registraron 342.035 AM (18,83\% de la población regional). Específicamente, en la comuna de Valparaíso hay 58.414 AM, que representan el 18,48\% de la población comunal total (INE, 2018). En el país se cuenta con una Política Integral de Envejecimiento Positivo 2012-2025, desarrollada por el Servicio Nacional del Adulto Mayor (SENAMA) del Ministerio de Desarrollo Social, que se constituye como una respuesta intersectorial a las necesidades de las personas AM (MINSAL, 2014).

La constatación de un problema nutricional en la población adulta mayor, en especial de aquella más vulnerable socioeconómicamente, llevó al gobierno de Chile a desarrollar un sistema de entrega de alimentos para este grupo poblacional, a través del denominado Programa de Alimentación Complementaria del AM (PACAM), cuyo objetivo ha sido contribuir a prevenir y tratar algunas carencias nutricionales, mantener o mejorar la funcionalidad física y psíquica, mejorar la detección y control de factores de riesgo e incentivar una mayor adherencia a las actividades del Programa de Salud del AM (MINSAL, 2016).

Sin embargo, se ha constatado también que los productos alimentarios del PACAM tienen poca variedad y luego de ser consumidos rutinariamente causan fatiga o aburrimiento y el AM pierde interés en ellos (Atalah y Masi, 2008), afectando la eficacia del programa. En el país no se han desarrollado iniciativas que apunten a revertir esta situación-problema. La experiencia que 
se implementó en un Centro de Salud Familiar (CESFAM) en la comuna de Valparaíso, que el artículo describe y analiza desde el aporte y perspectiva del diseño, se orientó a mejorar la eficacia del PACAM, en una escala local, buscando entregar preparaciones saludables listas para ser consumidas, especialmente orientadas a $\mathrm{AM}$ que, si bien son autovalentes, tienen dificultades para preparar sus alimentos. A través de la integración del diseño en este proyecto social multidisciplinar, se buscó que los AM participaran de las definiciones claves del sistema de comunicación, como un aspecto clave para el logro de sus objetivos.

En su idea central de cambiar una situación existente por una situación deseada (Simon, 1996), el diseño tiene un papel destacado en la generación de satisfactores de necesidades insatisfechas o desatendidas de personas que viven en sociedades con recursos limitados (Papanek, 2014). En este contexto, tres son las preguntas surgidas en la reflexión de la experiencia profesional de diseño en la implementación de un proyecto social y que intenta responder el artículo, a saber, ¿cuál es rol del diseñador en un "modelo social" de intervención profesional?, ¿cuál es el rol del usuario-participante, en este caso de los AM, en el proceso de co-diseño?, ¿cuál es el rol del diseño en el contexto social?

\section{Innovación y diseño social. Un marco teórico-conceptual para leer la experiencia profesional}

En el escenario de complejidad que representa el envejecimiento de la población, se han desarrollado nuevas formas de trabajo asociativo para abordar emergentes situaciones-problemas, involucrando a diversos actores como son las universidades, la comunidad afectada y a los organismos responsables de ejecutar las políticas públicas. Una de estas formas es la innovación social, que el Manual de Oslo (EUROSTAT y OCDE, 2006) define como "la introducción 
de un producto o proceso, un método de comercialización o un método organizativo nuevo o significativamente mejorado, en las prácticas internas de la empresa, la organización del lugar de trabajo o las relaciones exteriores" (p. 56). Esta definición es la más utilizada en el contexto empresarial; sin embargo, el Manual explicita que "la innovación puede estar presente en cualquier sector de la economía, incluyendo los servicios públicos tales como la salud o la educación" (EUROSTAT y OCDE, 2006, p. 23). La innovación social moviliza recursos sociales difusos (en términos de creatividad, habilidades, conocimiento y emprendimiento), constituyendo un importante motor de cambio, y podría convertirse en un poderoso promotor de formas de vida y producción sostenibles (Manzini et al., 2010). El diseño para la innovación social es definido por Manzini (2015) como "todo aquello que el diseño experto hace para activar, mantener y orientar los procesos de cambio social que llevan a la sostenibilidad" (Manzini, 2015, p. 81).

Papanek (2014), uno de los primeros teóricos del diseño, señala que las personas son diseñadores porque las actividades de la vida cotidiana necesitan del diseño, por tanto, el diseñador formado profesionalmente posee una responsabilidad social y de sostenibilidad ecológica al momento de proyectar. En este contexto, surgen nuevos paradigmas o dimensiones del diseño, Ledesma (2013) afirma que:

El diseño social aparece como una oposición al salvajismo del mercado que se hace evidente en una sociedad capitalista (p. 97). En esta perspectiva existen diversas miradas en las producciones del diseño: una tiene que ver con las intervenciones del diseño orientadas a desarrollos productivos que lo denomina diseño para el desarrollo, la segunda mirada hace referencia a la indeterminación semántica, término usado de maneras diferentes: social aparece como adjetivo destinado a caracterizar la vocación del diseño de ocuparse de toda la sociedad; como eufemismo para referirse a la acción a favor de los grupos desposeídos, minoritarios o marginales; apela a nociones vinculadas al desarrollo, es decir, al diseño orientado al mejoramiento de la calidad de vida de una sociedad, y por último postula una línea de investigación que analizaría los efectos del diseño sobre la sociedad. (p. 98) 
En el contexto de los países en vías en desarrollo y del cambio de paradigma de desarrollo que tuvo lugar a partir de la década de 1980, cuando una serie de comisiones internacionales dentro y fuera de la ONU ampliaron la definición de desarrollo para incluir su capacidad de crear bienestar humano y no solo una infraestructura económica, un proyecto de diseño puede aportar al desarrollo y, consecuentemente, a la satisfacción de necesidades específicas de un grupo de personas, como los AM. El Programa de las Naciones Unidas para el Desarrollo (PNUD) adoptó la idea de desarrollo humano, que considera cuestiones de cultura, igualdad social, salud, nutrición y educación, entre otros (Margolin, 2007). En este escenario, un desafío de la disciplina es la generación de un modelo de diseño de producto orientado a las necesidades sociales. Papanek propone que se deben desarrollar programas de diseño para las necesidades sociales que van desde aquellas generales de los países en desarrollo hasta aquellas asociadas a grupos poblacionales específicos, a saber, los ancianos, los pobres, los discapacitados, entre otros (Margolin y Margolin, 2012, p. 62). Desde esta perspectiva, existen dudas sobre si el mercado (invención social histórica) es el instrumento más adecuado para resolver los problemas que enfrentan las personas en la actualidad y en el futuro (Bonsiepe, 2012).

Margolin y Margolin (2012) proponen un "modelo social" de la práctica del diseño, el cual se basa en la intervención aplicada por los trabajadores sociales en colaboración con otros profesionales que se desempeñan en áreas de salud y educación, modelo que podría ser aplicado a países en desarrollo. Hacen énfasis que el modelo de diseño para el mercado centrado en la creación de productos para la venta y el modelo social para la satisfacción

\footnotetext{
1 Si bien el constructo "país en desarrollo" se encuentra en permanente debate, a propósito de la discusión en torno a la configuración del contenido del "desarrollo", entre economistas e instituciones internacionales preocupadas por el desarrollo de los países y regiones (Banco Mundial, 2020; CEPAL, 2020), con esta nominación se hace referencia a aquellos países que han superado un estado de subdesarrollo, contando con una buena calidad de vida y una buena cantidad de ingresos, pero no lo suficiente como para ser categorizados como un país desarrollado. Otras denominaciones equivalentes son "país intermedio" o "país en desarrollo".
} 
de las necesidades humanas no se configuran como "opuestos binarios" sino como "dos polos de un Continuum" (p. 64).

Para Manzini (2015):

la innovación social son ideas de nuevos productos, servicios y modelos que satisfacen las necesidades sociales y crean nuevas formas de colaboración en la actualidad (p. 14) y el diseño para la innovación social como todo aquello que el diseño experto hace para activar, mantener y orientar los procesos de cambio social que llevan a la sostenibilidad (p. 81), la diferencia entre diseño social y diseño para la innovación social tiende a diluirse conforme ambas actividades convergen y crean áreas en las que se solapan sus objetivos (de forma y productividad). El diseño social se orienta cada vez más hacia la innovación social, al recocer que esta es la única posibilidad de resolver los problemas que se abordan tradicionalmente. A su vez el diseño para la innovación social, ante la extensión de la crisis económica, se ve involucrado cada vez con mayor frecuencia en iniciativas que implican problemas socialmente sensibles. (p. 85)

En relación al argumento de Papanek, es necesario determinar si es el producto y el servicio y si los diseñadores socialmente responsables deben organizar sus intervenciones fuera del mercado convencional (Papanek citado en Margolin y Margolin, 2012, p. 68). En los países en desarrollo, la intervención del diseño social está limitada, ya que generalmente los recursos económicos disponibles para su inserción en proyectos sociales — que buscan aportar a la satisfacción de necesidades de los grupos poblacionales más vulnerados socioeconómicamente- son insuficientes y exclusivamente proporcionados por organismos públicos y organizaciones no gubernamentales sin fines de lucro, a través de fondos concursables. Aún en este escenario no óptimo, los proyectos sociales se constituyen en una herramienta que permite inducir un cambio a partir de las iniciativas de los actores que interactúan en un territorio o sector específico y tratan de revertir situaciones desventajosas que impiden el desarrollo local (Boisier citado en Baca y Herrera, 2016, p. 70).

La construcción de una respuesta disciplinaria y profesional socialmente apropiada, ha demandado la realización de estudios con el objetivo de 
comprender y apoyar el aporte del diseño en sociedades con recursos limitados, como lo que acontece en los países en desarrollo. La práctica del diseño en el campo de la intervención social se ve apoyada tanto por el desarrollo y la evaluación de métodos de diseño, como por la exploración académica de este campo (Jagtap y Larsson, 2018, p. 2664). En la práctica de intervención, los trabajadores sociales siguen un modelo de seis pasos que se configuran como: vinculación, valoración, planificación, implementación, evaluación y conclusión (Margolin y Margolin, 2012, p. 65). La importancia de este modelo es el trabajo colaborativo con otros profesionales. En el caso de la experiencia que se divulga en este artículo, el equipo multidisciplinario lo conforman profesionales de las áreas de diseño, alimentación saludable, geriatría y gastronomía, y los profesionales propios del Centro de Salud Familiar donde se llevó a cabo: terapeuta ocupacional y kinesiólogo.

La práctica del diseño participativo (DP) se constituye en uno de los aspectos importantes de la intervención social desde esta disciplina. En esta práctica hay dos conceptos claves, co-diseño y co-creación, desarrollados en países europeos, y que datan de los años setenta (Sanders y Stappers, 2008, p. 6). Si bien a menudo ambos conceptos son confundidos y/o tratados como sinónimos entre sí, Sanders y Simons (2009) realiza una distinción significativa. Define la co-creación como cualquier acto de creatividad colectiva, mientras que el concepto de co-diseño lo refiere a la creatividad colectiva aplicada específicamente al proceso de diseño. Según estas definiciones, el co-diseño es una instancia específica de co-creación (p. 1).

Entre diseñadores y miembros de la comunidad, el co-diseño es crucial para aminorar las diferencias culturales y sociales. En las últimas décadas, los diseñadores se han acercado cada vez más a los usuarios, lo cual ha influido en el panorama de la investigación en este ámbito. Para el diseñador es importante la participación de los usuarios (Sanders y Stappers, 2008, p. 5). 
Mattson y Wood (2014), plantean que el co-diseño ayuda tanto a los diseñadores como a los miembros de la comunidad. A los diseñadores les ayuda a conocer las necesidades, preferencias, circunstancias de la vida y aspiraciones de las personas que viven en comunidades postergadas, mejorando su comprensión de un entorno donde los recursos son limitados (p. 2). Ssozi-Mugarura et al. (2017) coinciden en que las actividades desarrolladas a partir del co-diseño mejoran la propiedad del proyecto en comunidades postergadas (p. 123).

Complementariamente, Mattson y Wood (2014) proponen nueve principios para un diseño eficaz para los países en desarrollo: 1. El co-diseño con personas en un contexto específico de país en desarrollo alienta la empatía del diseñador y empodera a las personas de escasos recursos; 2. Probar el producto en su contexto real es una parte esencial del diseño, no simplemente un paso final; 3. Importar tecnología sin adaptarla al contexto específico de un país en desarrollo es ineficaz e insostenible; 4. Tanto los individuos en contextos urbanos como rurales pueden beneficiarse de los esfuerzos de alivio de la pobreza; 5. Las mujeres y los niños se ven más afectados por los esfuerzos de alivio de la pobreza que los hombres; 6 . Técnicas de gestión de proyectos que se adaptan al contexto específico de un país en desarrollo permiten un proceso de diseño más eficaz y eficiente; 7. Los productos para un país en desarrollo tienen un mayor impacto cuando son contextualizados, desarrollados e implementados por equipos interdisciplinarios; 8. La cooperación con gobiernos y personas locales influyentes contextualiza y posibilita los planes de alivio de la pobreza; 9. Existen estrategias de distribución que pueden usarse para introducir productos en mercados de países en desarrollo (p. 2).

Existe un modelo tradicional para el DP que se basa en que los diseñadores se asocian con los usuarios y con las partes interesadas para realizar una co-creación. A menudo en el desarrollo de talleres se identifican las 
necesidades de los usuarios, los problemas, la tecnología y los productos existentes y se desarrollan nuevas soluciones. Sin embargo, se da por hecho que los participantes tienen las habilidades para contribuir al proceso de diseño y podrán trabajar junto a los diseñadores de una manera igualitaria. El modelo tradicional está muy simplificado, debe adaptarse a los escenarios donde se trabajará, ya que cada contexto es diferente, y en algunos casos no se logra una verdadera co-creación entre los diseñadores, los usuarios y las partes interesadas en un mismo nivel, sino que el diseñador tiene que tomar la iniciativa en las actividades de DP (Hussain et al., 2012).

Para Lee (2006), el DP se puede describir como la invitación de profesionales del diseño a legos, para que participen en el proceso de diseño, siendo la situación ideal la creación de soluciones colectivas a los problemas, especialmente para aquellos que están relacionados socialmente. Sin embargo, muchas prácticas de DP se convierten en otras formas de profesionalismo. Como ha definido Banham, "el profesionalismo es una forma de organizar la competencia, de reunir ciertas habilidades especializadas en un lugar y en una condición en la que puedan ayudar al resto de la sociedad, los profesionales están capacitados para orientarse hacia los problemas" (Banham citado en Lee, 2006, p. 13). Sin embargo, la forma en que resuelven los problemas se basa, tradicionalmente, en su interpretación de las necesidades de los demás, pero no en la formulación que hacen las propias personas de sus necesidades.

Otra perspectiva importante de destacar en este campo de preocupación es la propuesta de Manzini con el "mapa del modo del diseño", el cual se configura con dos dimensiones: 1. Un primer eje de "actores y competencias", que se desplaza del "diseño experto" al "diseño difuso", donde el diseño difuso es realizado por "inexpertos" y el diseño experto es puesto en marcha por profesionales de esta disciplina. 2. Un segundo eje de "motivaciones y expectativas" que va de "solucionar problemas" a "proporcionar sentido". 
A partir de este mapa, Manzini observa una diversidad de "culturas" emergentes de diseño, es decir, que han surgido gracias a un círculo virtuoso entre las nuevas ideas sobre la solución de problemas y la creación de sentido. Observa que la polarización tradicional entre los extremos tiende a menudo a desdibujarse y, en la actualidad, es cada vez más raro que esta separación se considere aceptable; ante los problemas complejos, hay diversas maneras de afrontarlos (Manzini, 2015, p. 51-56).

Desde el concepto de espacio social de Lefebvre, "espacio concreto" se puede definir como el espacio en el que vivimos y experimentamos, es decir, el mundo de las personas. El "espacio abstracto" es la abstracción espacial que suelen utilizar los expertos (Lefebvre citado en Lee, 2006, p. 2), de acuerdo a esta conceptualización el DP debe funcionar de una manera táctica, más que estratégica, para poder lidiar con la relación nunca establecida entre los espacios y los modos de DP a lo largo del tiempo (Lee, 2006).

Lee (2006), propone un nuevo enfoque y un plan de acción para los diseñadores y su forma de participación con la sociedad. El plan de acción (Figura 1), se desarrolla sobre la base de una herramienta analítica de DP con los tres modos de participación, a saber: Participación Pública, Participación con la Comunidad y Participación en Diseño. El rol del diseñador se convierte en un agente libre con conocimientos especiales de participación en el diseño, es decir, diseñar con personas y trabajar con los tres grupos de los tres modos de participación en el espacio abstracto (mundo de los expertos), el espacio concreto (mundo de las personas). Las actividades se ubican en un tejido multidireccional conformado por la intersección de los espacios abstracto y concreto, que tiene como objetivo fomentar una mayor colaboración y tácticas para ofrecer diferentes formas de participación en diferentes espacios operativos (Lee, 2006, p. 14). 


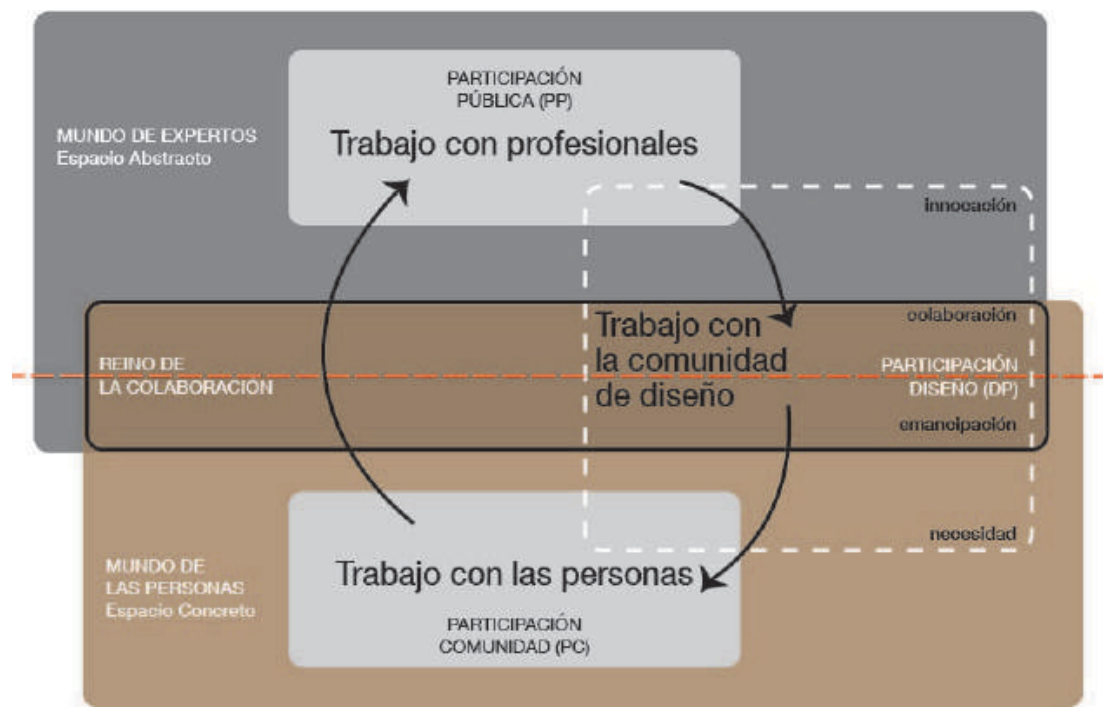

Figura 1. Plan de acción de las tácticas de participación en el diseño: más colaboraciones entre personas de diferentes espacios operativos. fuente: Lee (2006).

\section{Sujetos y métodos}

El método DP de acuerdo con Lee (2006), quien propone un nuevo enfoque y plan de acción sobre el trabajo de los diseñadores con la sociedad, establece tres modos de participación: Participación Pública, Participación con la Comunidad y Participación en Diseño. En el caso de la experiencia objeto de preocupación de este artículo, el DP comprendió siete acciones, divididas en tres etapas.

La primera etapa contempló tres acciones (Figura 2): una reunión de coordinación con el equipo de profesionales del CESFAM Reina Isabel II; 
una presentación del proyecto por el equipo de investigadores a los AM del Programa Más AM Autovalentes para invitarlos a participar; y una entrevista estructurada para caracterizar a los AM y recibir su firma del consentimiento informado para su participación en el estudio. Se entrevistaron a 18 AM, 16 mujeres $(88,8 \%)$ y 2 hombres $(11,1 \%)$, de edades comprendidas entre 61 y 79 años, que viven en el sector del cerro O`Higgins en la comuna de Valparaíso. Un 70\% de los AM tiene menos de 12 años de estudio (escolaridad obligatoria en Chile en la actualidad) y solo un $11 \%$ posee estudios técnicos-profesionales. La mayoría son pensionados/as y reciben un ingreso que no sobrepasa los 200.000 CLP (US 286) mensuales. La mayoría vive en hogares con 3,4 personas en promedio, un 66,6\% actúa como jefe de hogar y es el principal sostenedor económico de sus familias. En relación al estado civil, el 44,4\% señala estar casado o conviviendo con su pareja y el 55,55\% no tiene pareja (soltero, viudo o divorciado). Finalmente son 15 de estos AM, los que aceptaron participar en el proyecto social y en la experiencia de DP.

Si entendemos el nivel educativo alcanzado como un reflejo del nivel socioeconómico de una persona, su anterior vinculación al mercado laboral y su acceso al sistema de protección social, es razonable pensar que las personas sin educación o que solo alcanzaron la educación primaria vivieron una trayectoria laboral que las dejó en una situación de mayor vulnerabilidad ante la discapacidad en la vejez (CEPAL, 2017). 
Bastías Castillo, R., Lutz Riquelme, M., Siefer Navas, I., Gaete Fernández, R. / Aplicación del Diseño Social en una experiencia local orientada al mejoramiento de políticas públicas de envejecimiento poblacional, Valparaíso-Chile

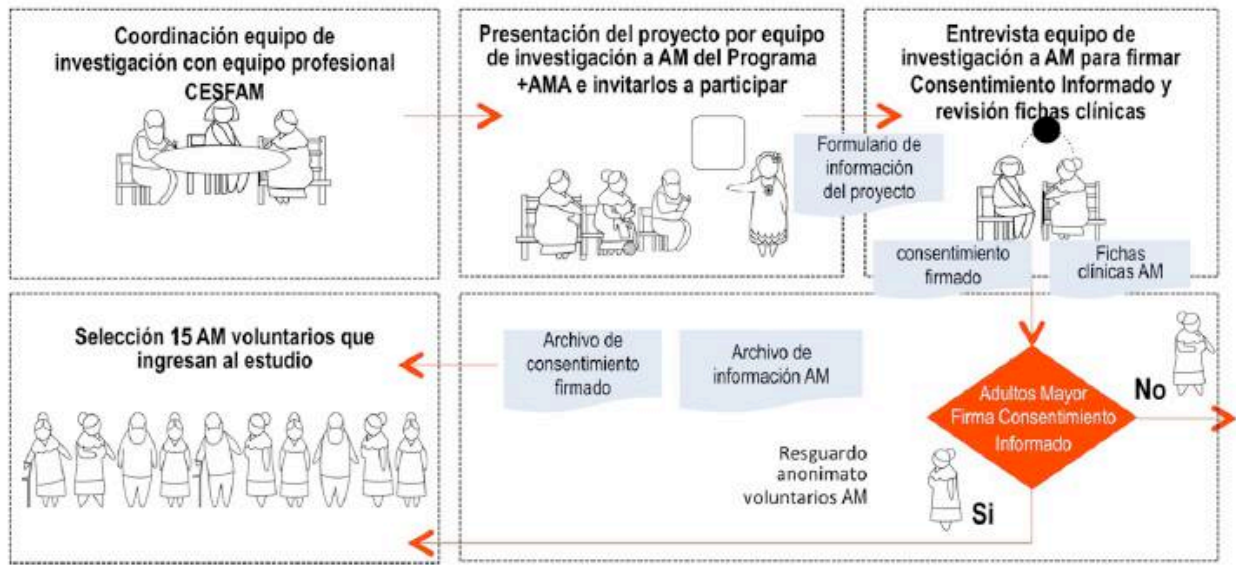

Figura 2. Primera Etapa de Diseño Participativo. Fuente: elaboración Propia.

La segunda etapa comprendió la realización de dos talleres de DP, organizados por el equipo de investigación con los profesionales del CESFAM para recoger la opinión de los AM sobre el material de comunicación: marca del proyecto, etiquetas y envases de las preparaciones alimentarias. El proceso de investigación fue documentado a través de las notas de los AM y fotografías de la ejecución de los talleres.

En el taller I (Figura 3) los participantes, orientados por el equipo de investigadores, conocieron los objetivos de cada sesión del taller de DP. Se los alentó a expresarse a través del diálogo y con notas escritas sobre sus opiniones de los diseños desarrollados propuestos. 


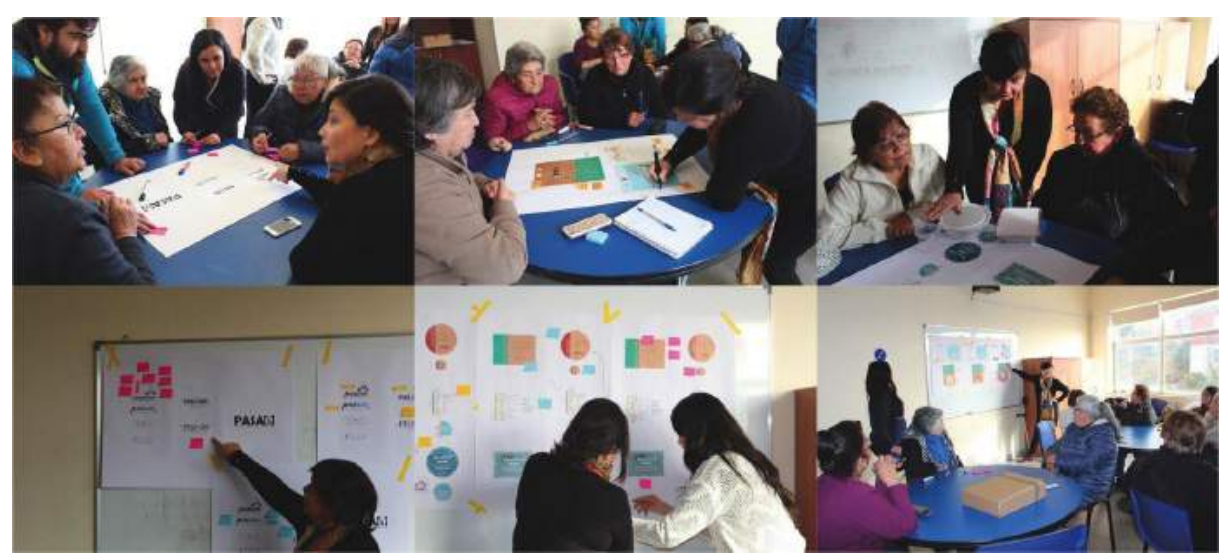

Figura 3. Taller de diseño participativo I. Fuente: elaboración propia.

Taller II: selección de diseños definitivos. Se presentaron los prototipos de diseño que incorporaron las opiniones emitidas por los AM en el taller I. El grupo de AM votó por la propuesta preferida y se realizó la selección de aquella que recibió la mayor cantidad de votos (Figura 4). 
Bastías Castillo, R., Lutz Riquelme, M., Siefer Navas, I., Gaete Fernández, R. / Aplicación del Diseño Social en una experiencia local orientada al mejoramiento de políticas públicas de envejecimiento poblacional, Valparaíso-Chile

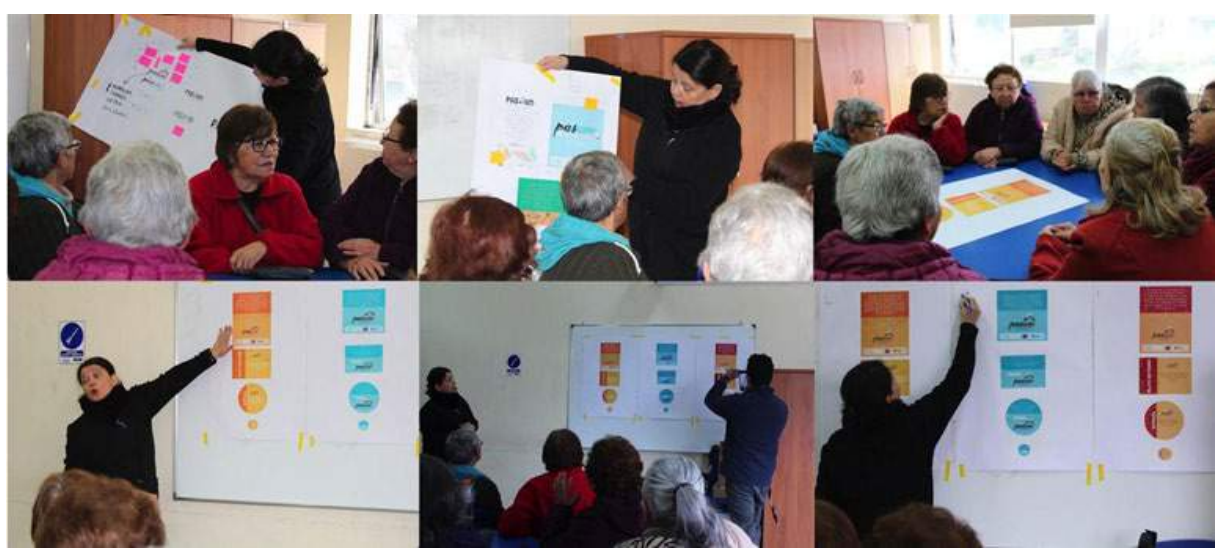

Figura 4. Taller de diseño participativo II. Fuente: elaboración propia.

Los talleres variaron en duración de una, a una y media hora, de acuerdo con el tiempo que se disponía para el uso de la sala multiuso facilitada para este efecto. Al final de cada taller, se recolectaron todas las opiniones de diseño expresadas por los AM. Finalmente, las decisiones de diseño fueron tomadas colectivamente tanto por los investigadores como por los AM participantes.

La tercera etapa consistió en la entrega de las preparaciones alimentarias saludables a los AM participantes. El ensayo, de tipo piloto, incluyó diferentes preparaciones culinarias elaboradas por un chef. Las preparaciones se entregaron dos veces por semana por tres meses (septiembre a diciembre de 2018). En esta etapa se les acompañó, registrando sus impresiones de manera de ajustar el sistema diseñado para optimizarlo. 
Una vez terminado el período de entrega de las preparaciones alimentarias, se realizó la ceremonia de cierre del proyecto, y se hizo entrega a los AM y personal del CESFAM de un Recetario con las preparaciones ensayadas.

\section{Hallazgos}

El trabajo de campo fue realizado por un equipo interdisciplinar de investigación, más los profesionales del CESFAM a cargo del Programa Más AM Autovalentes y se ejecutó entre marzo y diciembre de 2018. El trabajo permitió establecer un espacio de colaboración (Lee, 2006) entre el equipo de expertos y la comunidad de AM, donde se consulta la opinión a los AM de las propuestas de diseño sobre los prototipos (la marca y el sistema de etiquetas para los envases que contendrán las preparaciones alimentarias) y se empodera a los AM participantes con el proyecto. Las actividades de DP actuaron como un facilitador que potenció las habilidades sociales de los AM. De acuerdo con Hussain et al. (2012), desde esta perspectiva, el profesional tiene un rol de colaborador y de facilitador que proporciona los recursos, más que de un experto y/o consultor para la comunidad, Manzini propone el "mapa del modo del diseño" ya que en la actualidad existen nuevas ideas para la solución de los problemas y la creación de sentido, y la polarización de los extremos entre el "diseño experto" y "diseño difuso" cada vez tiende a desdibujarse (Manzini, 2015). Así la comunidad se convierte en una participante activa en el proceso de su propio desarrollo. (Zimmerman en Silva y Martínez, 2004, p. 30)

En los dos talleres realizados con los AM hubo una actitud muy positiva de los participantes, que se tradujo en proactividad, alta colaboración y disposición a retroalimentar las propuestas realizadas por el equipo de diseño, siendo estas instancias de co-diseño (Sanders y Simons, 2009) con la comunidad de AM. 


\section{Taller I Diseño Participativo}

En la consulta sobre la marca del proyecto se presentaron tres propuestas, explicando a los AM el significado de la marca, qué contenía el nombre Preparaciones Alimentarias Saludables para el Adulto Mayor "PASAM", qué es signo distintivo (imagen visual-dibujo de una pareja de AM activos) que permitiría identificar el proyecto. Se realizaron cinco preguntas gatilladoras con la idea de generar un diálogo y recoger la opinión de los AM sobre las propuestas de marca: ¿qué opina del nombre?, ¿qué opina del diseño?, ¿qué opina de los dibujos?, ¿qué opina de los colores?, ¿qué opina del tamaño de las letras?

Como se observa en la Figura 5, de las tres propuestas presentadas a los AM, sobre dos de las marcas (" $\mathrm{A}$ " $\mathrm{y}$ "B"), los AM expresaron su opinión y sobre una de ellas ("C $\mathrm{C}^{\prime}$ ) no hubo manifestación ni a favor o contra de la propuesta de diseño, es decir, fueron totalmente indiferentes. Los atributos positivos de la marca " $\mathrm{A}$ " que más destacan los AM son los siguientes: el diseño novedoso, el nombre, los dibujos, los colores Ilamativos y sobre los aspectos que se debieran ajustar son los siguientes: letras más grandes, claridad de la letra.
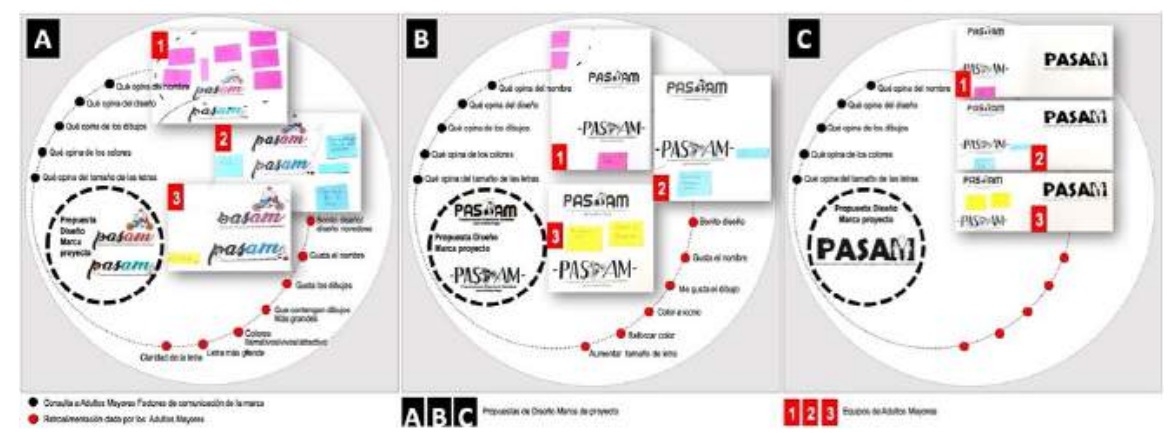

Figura 5. Propuesta de marca. Fuente: elaboración propia. 
En la consulta sobre el sistema de etiquetas para los envases de las preparaciones alimentarias se presentaron tres propuestas, indicando a los AM la función de las etiquetas, la ubicación que tendrían en los envases de las preparaciones alimentarias y que se estructuraba en un sistema de etiquetas para la entrada, el plato principal y el postre. Como se observa en la Figura 6, se presentaron tres propuestas y se realizaron cuatro preguntas que tienen la finalidad de recoger la opinión de los AM sobre los sistemas de etiquetas: ¿qué opina del diseño?, ¿qué opina de los colores?, ¿qué opina del tamaño de las letras?, ¿qué opina de los dibujos?

Los elementos de la propuesta "A" destacados son: el diseño es más llamativo, colores llamativos, usar dibujos de la otra marca, dividir el plano de color, realzar contraste y agregar ingredientes. De la propuesta "B" solo destacaron la diagramación. De la propuesta "C", los AM opinaron que debía cambiar a un tono más claro, no les gustan los distintos colores, es necesario agregar la marca, usar letra clara y legible, asociar los dibujos con el contenido indicado en la etiqueta.



Figura 6. Sistema de etiquetas para envases de preparaciones alimentarias. Fuente: elaboración propia. 
En la consulta sobre la etiqueta para la caja contenedora de los envases de las preparaciones alimentarias, se presentaron las propuestas y se procedió indicar a los AM su función y la ubicación que tendrían en la caja que contendría las preparaciones alimentarias. Como se observa en la Figura 7, se presentaron tres propuestas y realizaron cuatro preguntas a los AM sobre las propuestas de etiquetas: ¿qué opina del diseño?, ¿qué opina de los colores?, ¿qué opina del tamaño de las letras?, ¿qué opina de los dibujos?

De la propuesta "A", los AM destacaron: es muy pálido, le falta color, estas letras podrían ser negras, faltan dibujos con color, color muy suave, mantener íconos, logos como esta propuesta "A". De la propuesta "B" destacaron el color celeste-tonalidades, aclarar color, colorear los dibujos, usar dibujos de alimentos, agrandar logos y usar logos con transparencias.

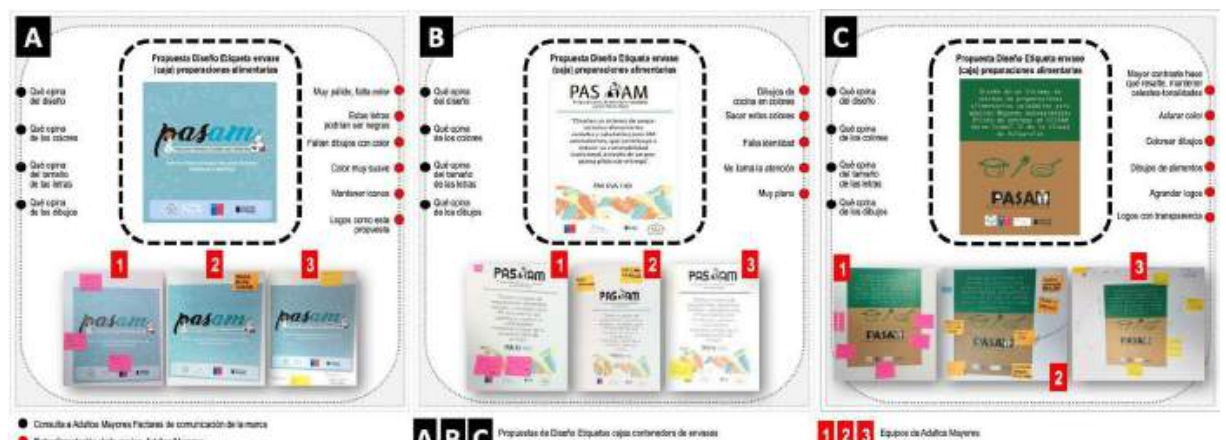

Figura 7. Etiqueta para caja de envases. Fuente: elaboración propia. 


\section{Taller II Diseño Participativo}

El segundo taller consistió en la consulta sobre el sistema de etiquetas de los envases de preparaciones alimentarias saludables para el proyecto. Se presentaron tres propuestas que contenían las observaciones más importantes recogidas de los AM en el taller I Diseño Participativo. Este taller se realizó con todos Ios AM que habían asistido a la convocatoria del taller I, generando nuevamente un espacio de colaboración (Lee, 2006), donde se les informó a los AM que el equipo de diseño en el espacio de expertos (Lee, 2006) había incorporado sus observaciones a las tres propuestas definitivas.

El equipo de diseño explicó a los AM la dinámica del taller, consistente en que cada AM presente votaría por una de las tres propuestas que le parecía más apropiada para el sistema de etiquetas. En la Figura 8 se observan dos imágenes: a la derecha se encuentran las tres propuestas elaboradas por el equipo de diseño y a la izquierda el resultado de la votación emitida por los AM. Cuando se consulta a los AM sobre el diseño, la investigación se enriquece y para los "usuarios" el diseño toma sentido. En este caso se presentaron propuestas desarrolladas y los diseñadores tomaron el rol de facilitadores para que los AM opinaran y decidieran sobre la propuesta que ellos consideraban que era la más apropiada e indicaran los cambios que debían realizar los diseñadores.

De acuerdo Hussain et al. (2012), el modelo tradicional de DP se ha adaptado al contexto y condiciones que se tenía para trabajar en el CESFAM, en relación con el uso de la sala multiuso y el tiempo disponible para el desarrollo de la actividad, ya que consideramos que con los AM se debería haber dispuesto de mayor cantidad de horas para haber trabajado de manera igualitaria los usuarios (AM) y diseñadores con el propósito de haber enriquecido el espacio de colaboración entre los actores participantes en el proyecto. 
Bastías Castillo, R., Lutz Riquelme, M., Siefer Navas, I., Gaete Fernández, R. / Aplicación del Diseño Social en una experiencia local orientada al mejoramiento de políticas públicas de envejecimiento poblacional, Valparaíso-Chile

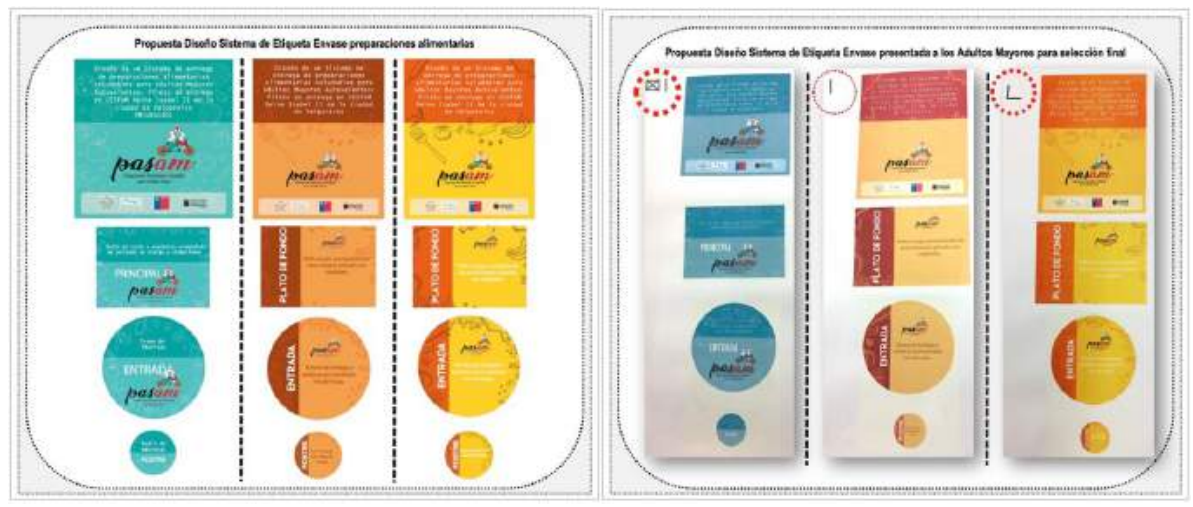

Figura 8. Selección final del sistema de etiquetas. Fuente: elaboración propia.

\section{Puesta en marcha del piloto de entrega de las} preparaciones alimentarias a los AM

El sistema de etiquetas acordado fue aplicado a los envases que contenían las preparaciones alimentarias saludables. Como se observa en la Figura 9, cada preparación (entrada, plato principal, postre) se entregó a los AM en cajas individuales. 


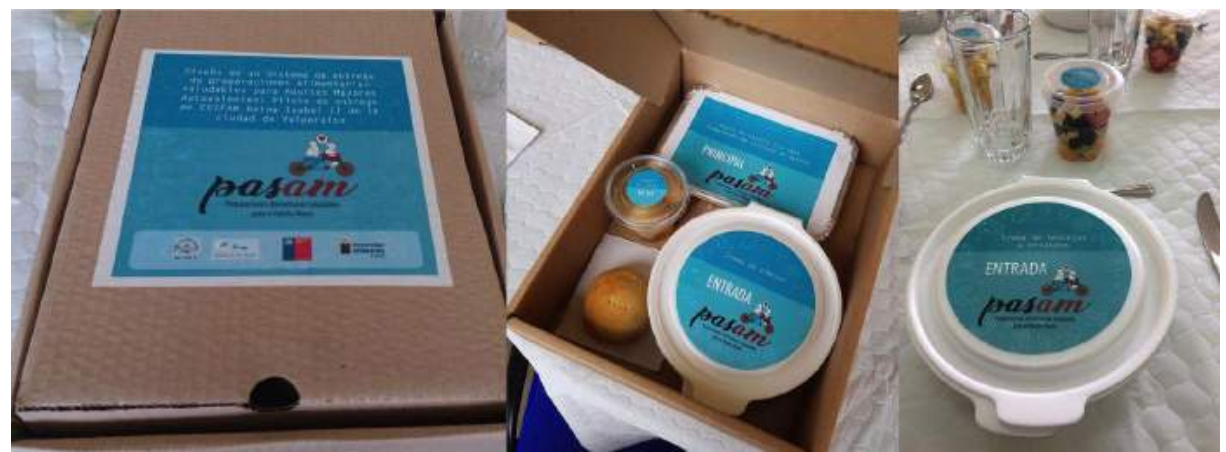

Figura 9. Aplicación de Sistema de etiquetas. Fuente: elaboración propia.

Los AM acudieron sistemáticamente a la entrega de las preparaciones alimentarias, mostrando una alta asistencia a las sesiones de entrega. La Figura 10 muestra una sesión de almuerzo. Además, se generó alta socialización entre los participantes en el proyecto, lo que significa que proyectos de esta naturaleza permite a los AM dar uso a su tiempo en una actividad social. La Encuesta Nacional del Uso del Tiempo, ENUT 2015, muestra el tiempo de ocio y vida social se reduce con el aumento de la edad de las personas. Tiempo promedio sobre personas sobre 66 años y más en un día tipo dedicado a actividades de ocio y vida social, en hombres es 5,79 y mujeres 5,61 horas (INE, 2015). 


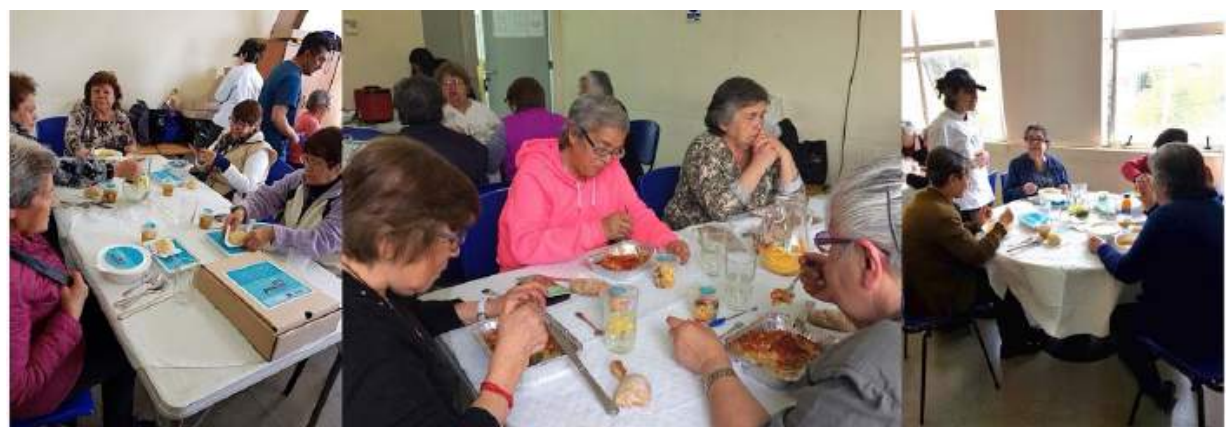

Figura 10. Adultos Mayores consumiendo las preparaciones alimentarias saludables. Fuente: elaboración propia.

En la última sesión de entrega de las preparaciones alimentarias a los AM, el chef diseñó una forma diferente de entrega (Figura 11): se dispuso el mobiliario de la sala multiuso de manera lineal, con el propósito de ubicar las preparaciones a la vista, y se aplicó un sistema de autoservicio. Se ofrecieron tres alternativas de plato principal y dos postres, para que ellos degustaran y eligieran. Esta modalidad fue gratamente recepcionada por los AM, quienes expresaron que se sentían muy agradecidos por participar en todas las actividades que contempló el proyecto.

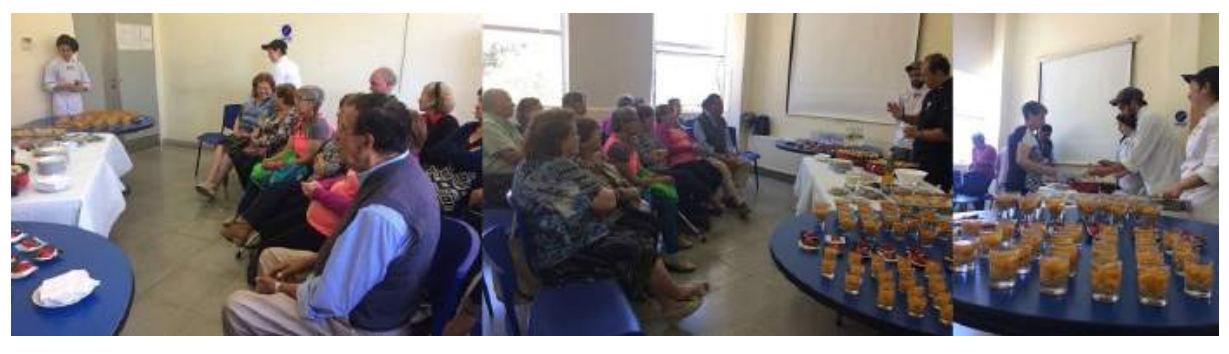

Figura 11. Última sesión de entrega de las preparaciones alimentarias saludables. Fuente: elaboración propia. 
La última acción del trabajo de campo fue la ceremonia de cierre del proyecto. Como se observa en la Figura 12, consistió en pequeñas intervenciones de los AM, profesionales del proyecto y directivos de la Universidad de Valparaíso. En ella se informó a los AM la importancia del diseño participativo, argumentando que para que estos proyectos se implementen de manera óptima, los participantes, profesionales, investigadores y las instituciones a cargo del desarrollo de los productos y servicios deben estar dispuestos a trabajar de forma colaborativa, es decir, el trabajo con las personas, los profesionales y el trabajo de comunidad de diseño en un espacio de colaboración (Lee, 2006).

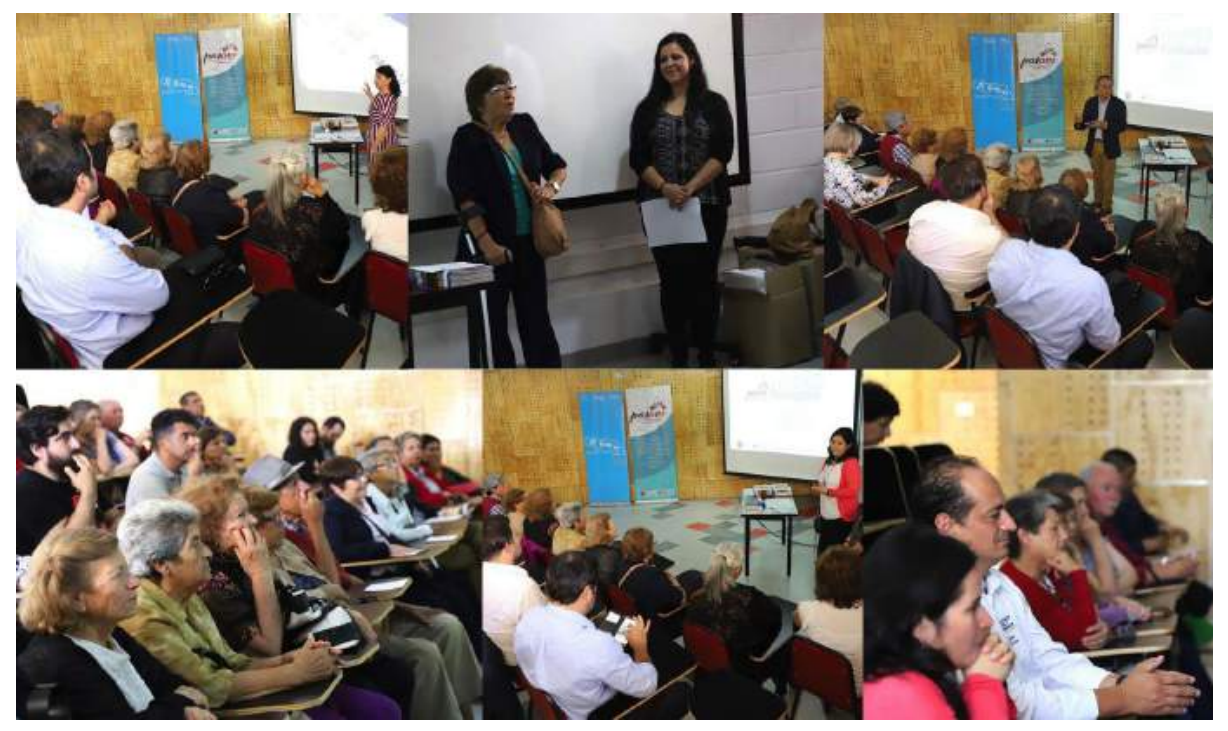

Figura 12. Ceremonia de cierre del proyecto Fuente: fotografías de Constanza Castillo Montroni. 
En la ceremonia de cierre del proyecto se hizo entrega de un diploma de participación y un recetario que contenía todas las preparaciones alimentarias que los AM recibieron durante el desarrollo del proyecto (Figura 13). Desde el punto de vista del DP, el reconocimiento del aporte y la participación voluntaria de los $\mathrm{AM}$ en el proyecto es un hecho que debe ser reconocido por el equipo de investigación y la ceremonia de cierre tuvo este fin, de agradecimiento a los AM, a los directivos del CESFAM Reina Isabel II y al equipo de profesionales del programa Más AM Autovalentes.

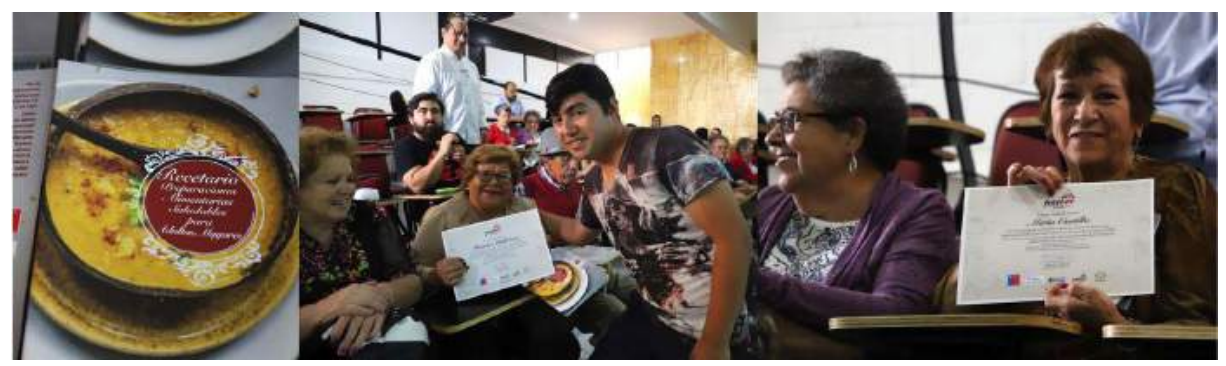

Figura 13. Entrega de recetario y diplomas a Adultos Mayores. Fuente: fotografías de Constanza Castillo Montroni.

De acuerdo con los principios propuestos por Mattson y Wood (2014) para un DP eficaz en los países en desarrollo, se puede afirmar que las actividades realizadas se ejecutaron en un contexto específico, se produjo empatía y generó un empoderamiento de los AM. Durante todo el desarrollo del proyecto se probaron los productos, toda la gestión fue adaptada al contexto específico, el proyecto fue desarrollado e implementado por un equipo multidisciplinar, con colaboración entre el equipo de la Universidad de Valparaíso, directivos del CESFAM y los profesionales del programa Más AM Autovalentes que permitió su éxito. La investigación desarrollada permitió comprender las características de un proyecto de diseño social o de diseño para la innovación social, como 
expresa Manzini (2015) que "el diseño social se orienta cada vez más hacia la innovación social, al reconocer que esta es la única posibilidad de resolver los problemas, que ante la extensión de la crisis económica, se ve involucrado cada vez con mayor frecuencia en iniciativas que implican problemas socialmente sensibles" (Manzini, 2015, p. 85). En un país en desarrollo, en un contexto y un grupo de personas con características específicas. La experiencia piloto desarrollada es posible de replicar en otros Centros de Salud.

\section{Conclusiones}

El trabajo realizado permitió identificar tres factores críticos para el diseño participativo:

1. El rol del diseñador en un "modelo social" de intervención profesional. El trabajo se enmarcó en un "modelo social" (Margolin y Margolin, 2012) de la práctica del diseño y, de acuerdo con Manzini, el diseño debe orientarse hacia la innovación social para la resolución de problemas socialmente sensibles (Manzini, 2015). El diseño no ha sido el foco exclusivo en la ejecución del proyecto de investigación, sino que han participado otros profesionales que, desde sus disciplinas, trabajaron colaborativamente en el desarrollo del proyecto. El rol del diseñador es ser un agente con conocimientos especiales que actúa en el espacio abstracto (mundo de los expertos), el espacio concreto (mundo de las personas) y participa con la sociedad a través de los tres modos de participación: Participación Pública, Participación con la Comunidad y Participación en Diseño (Lee, 2006). El diseñador debe ser un facilitador y desarrollar la práctica de la colaboración en diseño que tiene como objetivo fomentar el proceso del co-diseño (Lee, 2006) para que las personas expresen su creatividad, opinen y participen activamente del proceso de diseño. Cuando el diseñador pregunta a las personas sobre el proceso diseño, la investigación se nutre y amplía. 
El diseño se vuelve significativo para los "usuarios", participantes en un proyecto social.

2. El rol del usuario-participante (AM) en el proceso de co-diseño. En el trabajo de campo desarrollado bajo el método diseño participativo se constató que las personas que se ubican en el espacio concreto y aportan sus conocimientos y necesidades en el espacio de colaboración (Lee, 2006), tienen la capacidad de observar y dar su opinión frente a un objeto de diseño y, como tal, distinguen factores de diseño y comunicación que están presentes en una pieza de diseño. El usuario cumple un rol fundamental de participación y de acuerdo con Manzini "un número creciente de personas al experimentar las dificultades de individualización extrema, deciden cooperar para resolver problemas o para plantear alternativas. Es decir, redescubren el poder de hacer cosas juntos" (Manzini, 2015, p.112), y de esta forma contribuyen al proceso de co-diseño, que ayuda a mejorar y ampliar las propuestas de diseño realizadas por el equipo experto. El usuario es un factor clave en el proceso de co-diseño, es un actor que tiene la capacidad de opinar y reflexionar sobre sus necesidades, en un espacio de colaboración con los otros actores partícipes del proyecto.

3. El rol del diseño en el contexto social. En el campo de la participación en diseño, el rol del diseño es dar respuesta a las necesidades especiales de los grupos específicos de la sociedad (AM) y, como propone Papanek (2014), transitar del diseño social al diseño para la innovación social con el propósito de desarrollar programas que den respuestas a problemas sociales cada vez más complejos (Manzini, 2015), sobre todo cuando el mercado no es el instrumento más apropiado para dar respuesta a los problemas que afectan a las personas (Bonsiepe, 2012). En el diseño en un contexto social debe hacer uso de métodos que se diferencien del trabajo tradicional del diseño, esto implica usar nuevas herramientas que permitan conectar a 
los profesionales con los "usuarios" y beneficiaros de los resultados del proyecto. El diseño participativo se configura como una herramienta que permite al equipo de profesionales intervenir en un contexto social específico con sus tres modos de participación: Participación Pública, Participación con la Comunidad y Participación en Diseño, en un espacio de intersección entre el espacio abstracto y espacio concreto que tienen como objetivo fomentar la colaboración (Lee, 2006) entre los profesionales y el grupo específico de la comunidad participantes en el proyecto.

\section{Agradecimientos}

Esta investigación ha sido financiada por el Programa de Mejoramiento Institucional PMI UVA 1401 "GERÓPOLIS: Modelo de educación, salud y territorio: enfrentando la desigualdad en la tercera edad de la Ciudad Valparaíso" de la Universidad de Valparaíso. Se agradece a los directivos del CESFAM Reina Isabel II, a los profesionales del Programa Más AM Autovalentes y a los AM que participaron en esta investigación.

\section{Referencias}

Apella, I., Packard, T., Joubert, C., y Zumaeta, M. (2019). Retos y oportunidades del envejecimiento en Chile. Santiago de Chile: Grupo Banco Mundial http://documents1.worldbank.org/curated/es/591471558704698806/pdf/ Retos-y-Oportunidades-del-Envejecimiento-en-Chile.pdf

Atalah, E. y Masi, C. (2008). Análisis de la aceptabilidad, consumo y aporte nutricional del programa alimentario del adulto mayor. Revista Médica de Chile, 136(4), 415-422. https://www.researchgate.net/ publication/41145700_Analisis_de_la_aceptabilidad_consumo_y_aporte_ nutricional_del_programa_alimentario_del_adulto_mayor 
Baca, N. y Herrera, F. (2016). Proyectos sociales. Notas sobre su diseño y gestión en territorios rurales. Convergencia, 72, 69-87. http://www.scielo. org.mx/scielo.php?script=sci_arttext\&pid=S1405-14352016000300069

Banco Mundial. (2020). El comercio al servicio del desarrollo. En la era de las cadenas de valor mundiales. Panorama general. https://openknowledge. worldbank.org/bitstream/handle/10986/32437/211457ovSP.pdf

Bonsiepe, G. (2012). Diseño y Crisis. Valencia: Campgràfic Editors.

CEPAL. (2017). Panorama Social de América Latina, 2016. https://www.cepal. org/es/publicaciones/41598-panorama-social-america-latina-2016

CEPAL.(2020).América Latina yel Caribeantela pandemia delCOVID-19. Efectos económicos y sociales. https://www.cepal.org/es/publicaciones/45337america-latina-caribe-la-pandemia-covid-19-efectos-economicos-sociales

EUROSTAT y OCDE. (2006). Manual de Oslo (3 ${ }^{\mathrm{a}}$ Ed.). http://www.itq.edu.mx/ convocatorias/manualdeoslo.pdf

Hussain, S., Sanders, E. B-N. y Steinert, M. (2012). Participatory Design with Marginalized People in Developing Countries: Challenges and Opportunities Experienced in a Field Study in Cambodia. International Journal of Design, 6(2), 91-109. https://www.researchgate.net/profile/ Martin-Steinert/publication/263162024_Participatory_Design_ with_Marginalized_People_in_Developing_Countries_Challenges_ and_Opportunities_Experienced_in_a_Field_Study_in_Cambodia/ links/544159480cf2a6a049a57676/Participatory-Design-withMarginalized-People-in-Developing-Countries-Challenges-andOpportunities-Experienced-in-a-Field-Study-in-Cambodia.pdf 
Instituto Nacional de Estadística. (2015). Encuesta nacional del uso del tiempo 2015. Síntesis de resultados. https://www.ine.cl/docs/default-source/usodel-tiempo-tiempo-libre/publicaciones-y-anuarios/publicaciones/sintesisresultados-actividades-personales-enut.pdf?sfvrsn=fd9a7cea_6

Instituto Nacional de Estadística (2018). Memoria del Censo 2017. Santiago de Chile. https://www.censo2017.cl/memoria/descargas/memoria/libro_ memoria_censal_2017_final.pdf

Jagtap, S. y Larsson, T. (mayo, 2018). Design and frugal innovations: Three roles of resource-poor people (Proceedings of the International Design Conference). The Design Society Croacia, 2657-2668. https://www. designsociety.org/multimedia/publication/308e56d0ab2aec1a7879093cc 446bd960b1a00cb0b3b2e0215a920f2f1be97e212919bab1525938610. pdf

Ministerio de Salud de Chile. (2014). Programa Nacional de Salud de las personas Adulto Mayor, MINSAL 2014. https://www.minsal.cl/sites/default/ files/files/Borrador\%20documento\%20Programa\%20Nacional\%20de\%20 Personas\%20Adultas\%20Mayores-\%2004-03_14.pdf

Ministerio de Salud de Chile. (2016). Norma Técnica de los Programas Alimentarios (PACAM). https://www.minsal.cl/wp-content/uploads/2015/ 09/2016.04.20-Norma-T\%C3\%A9cnica-Prog.-Alimentarios-aprobadapor-Jur\%C3\%ADdica.pdf

Lee, Y. (2006). Design Participation Tactics: Redefining User Participation in Design (International Conference). Design Research Society Lisbon. IADE. http://citeseerx.ist.psu.edu/viewdoc/download?doi=10.1.1.567.8959\&rep =rep $1 \&$ type=pdf 
Ledesma, M. (2013). Cartografía del Diseño Social. Aproximaciones conceptuales. Anales del IAA, 43(1), 97-106. https://www.google.com/sea rch?q=Ledesma $\% 2 C+$ M. $+(2013)$. + Cartograf $\% C 3 \% A D a+$ del + Dise $\%$ C3\%B 10+Social.+Aproximaciones+conceptuales.+Anales+del+IAA\%E2\%80\%8 B $\% 2 C+\% E 2 \% 80 \% 8 B 43(1) \% 2 C+97-106 . \& r l z=1 C 5 C H F A \_e n C L 844 C L 844$ \&oq $=$ Ledesma $\% 2 \mathrm{C}+$ M.+(2013).+Cartograf\%C3\%ADa+del+Dise $\%$ C3\%B 10+Social.+Aproximaciones+conceptuales.+Anales+del+IAA\%E2\%80\%8 $\mathrm{B} \% 2 \mathrm{C}+\% \mathrm{E} 2 \% 80 \% 8 \mathrm{~B} 43(1) \% 2 \mathrm{C}+97-106 .+\& a q \mathrm{~s}=$ chrome..69i57.993j0j15 \&sourceid=chrome\&ie $=$ UTF-8

Manzini, E., Penin, L., Gong, M., Cipolla, C., M'Rithaa, M. \& Mendoza, A. (2010). The DESIS network: design and Social innovation for Sustainability. J. Design Strategies, 4. https://issuu.com/journalofdesignstrategies/docs/ the_journal_of_design_strategies_vo_3d28703899b84f

Manzini, E. (2015). Cuando todos diseñan. Una introducción al diseño para la innovación social. Madrid, España: Experimenta Editorial.

Margolin, V. (2007). Design for Development: Towards a History. Design Studies, 28, 111-115.https://www.sciencedirect.com/science/article/pii/ S0142694X06000809?via\%3Dihub

Margolin, V. y Margolin, S. (2012). Un "modelo social" de diseño: cuestiones de práctica e investigación. Revista KEPES, 9(8), 61-71. http://190.15.17.25/ kepes/downloads/Revista8_4.pdf

Marmot, M., Friel, S., Bell, R., Houweling, T.A., y Taylor, S. (2008). Closing the gap in a generation: health equity through action on the social determinants of health. Lancet, 372(9650), 1661-1669. https://www.sciencedirect.com/ science/article/abs/pii/S0140673608616906 
Mattson, C. y Wood, A. (2014). Nine Principles for Design for the Developing World as Derived From the Engineering Literature. J. Mechanical Design, 136, 1-15. http://mechanicaldesign.asmedigitalcollection.asme.org/article. aspx?articleid $=1888742$

Organización de las Naciones Unidas. (2017). World Population Prospects: The 2018 Revision, Key Findings and Advance. New York, USA: Department of Economic and Social Affairs, Population Division. https://www.un.org/ development/desa/publications/world-population-prospects-the-2017revision.html

Organización Mundial de la Salud. (2015). Ageing and Health, Fact Sheet No 404. http://www.who.int/news-room/fact-sheets/detail/ageing-and-health

Papanek, V. (2014), Diseño para un mundo real. Barcelona, España: Pol.len edicions.

Sanders, E.B-N. y Stappers, P.J. (2008). Co-creation and the new landscapes of design. Taylor \& Francis, 4(1), 5-18. https://www.researchgate.net/ publication/235700862_Cocreation_and_the_New_Landscapes_of_ Design

Sanders, E.B-N. y Simons, G. (2009). A social vision for value co-creation in design. Technology Innovation Managment Review, 1-5. https://timreview. ca/article/310

Silva, C. y Martínez, M.L. (2004). Empoderamiento: Proceso, Nivel y Contexto. Psykhe (Santiago), 13(2), 29-39. https://www.redalyc.org/ pdf/967/96713203.pdf

Simon, H. A. (1996). The Sciences of the Artificial. Cambridge: MIT Press. https://monoskop.org/images/9/9c/Simon_Herbert_A_The_Sciences_of_ the_Artificial_3rd_ed.pdf 
Bastías Castillo, R., Lutz Riquelme, M., Siefer Navas, I., Gaete Fernández, R. / Aplicación del Diseño Social en una experiencia local orientada al mejoramiento de políticas públicas de envejecimiento poblacional, Valparaíso-Chile

Ssozi-Mugarura, F., Blake, E. y Rivett, U. (2017). Codesigning with communities to support rural water management in Uganda. CoDesign, 13(2), 110-126. https://www.tandfonline.com/doi/pdf/10.1080/15710882.2017.1310904? needAccess=true

Welti, C. (2013). Política social y envejecimiento, Papeles de Población, 19(77). http://www.scielo.org.mx/scielo.php?script=sci_arttext\&pid=S140574252013000300005

Cómo citar: Bastías Castillo, R., Lutz Riquelme, M., Siefer Navas, I., Gaete Fernández, R. (2021). Aplicación del Diseño Social en una experiencia local orientada al mejoramiento de políticas públicas de envejecimiento poblacional, Valparaíso-Chile. Revista KEPES, 18(23), 75-109. https://doi.org/10.17151/kepes.2021.18.23.4 